\title{
Current Percolation in Medium with Boundaries under Quantum Hall Effect Conditions
}

\author{
M. U. Malakeeva ${ }^{1}$ and V. E. Arkhincheev ${ }^{2}$ \\ ${ }^{1}$ Department of Physics and Mathematics, Zabaikalskii State Human Pedagogic University, Chita 681, Russia \\ ${ }^{2}$ Buryat Science Center, SB RAS, Ulan-Ude 670047, Russia
}

Correspondence should be addressed to V. E. Arkhincheev, varkhin@mail.ru

Received 12 October 2011; Accepted 30 November 2011

Academic Editor: Mohindar S. Seehra

Copyright ( ) 2012 M. U. Malakeeva and V. E. Arkhincheev. This is an open access article distributed under the Creative Commons Attribution License, which permits unrestricted use, distribution, and reproduction in any medium, provided the original work is properly cited.

The current percolation has been considered in the medium with boundaries under quantum Hall effect conditions. It has been shown that in that case the effective Hall conductivity has a nonzero value due to percolation of the Hall current through the finite number of singular points (in our model these are corners at the phase joints).

\section{Introduction}

One of the basic issues of the study of current percolation in the heterogeneous medium is the problem to evaluate the effective conductivity $\sigma_{e}$. It is determined as an aspect ratio of average values of electric current $\vec{J}=\langle\vec{j}\rangle$ to electric field $\vec{E}=\langle\vec{e}\rangle$ :

$$
\vec{J}=\sigma_{e} \vec{E}
$$

In two-dimensional case the exact results for the effective conductivity have been obtained due to the dual symmetry of equations for the constant current. The Keller's [1] theorem and general approach developed in Dykhne works [2, 3] belong to them. It was shown that the effective conductivity of two-phase medium with $\sigma_{1}$ and $\sigma_{2}$ being the conductivities of phases at the percolation threshold (at equal phase concentrations) is

$$
\sigma_{e}(0)=\sqrt{\sigma_{1} \sigma_{2}}
$$

The duality relation for the effective conductivity at the arbitrary concentrations of phases has been deduced:

$$
\sigma_{e}(\varepsilon) \sigma_{e}(-\varepsilon)=\sigma_{1} \sigma_{2}
$$

Here $\varepsilon=x-x_{c}$ is the deviation from the percolation threshold $x_{c}=1 / 2$. (The dual system is that which is different from initial one only in rearrangement of phases, while the geometrical location of phases remains the same.)

However, the same reasoning is not applicable to a study of the effective Hall conductivity in the heterogeneous twodimensional system under conditions of the quantum Hall effect: $\sigma_{x x}=0, \sigma_{x y}=$ const. As is known, in this case the electric current always flows perpendicular to the electric field [4]:

$$
\vec{j}=\sigma_{x y}[\vec{n}, \vec{e}]
$$

Here the $\vec{n}$ is unit vector directed perpendicular to the twodimensional plane along the magnetic field. That is why Joule heat always equals zero, when the current percolates at QHE conditions.

$$
Q=0
$$

that means that Hall phases are dissipativeless. Another feature is inherent to the current percolation process at QHE conditions as well. The point is that, instead of ordinary boundary conditions, that is, continuity of tangential components of electric field $e_{1 t}=e_{2 t}$ as well as continuity of normal components of electric current $j_{1 n}=j_{2 n}$, the new boundary conditions arise:

$$
j_{1 n}=j_{2 n}=0
$$


$\sigma_{x y}^{(1)}$

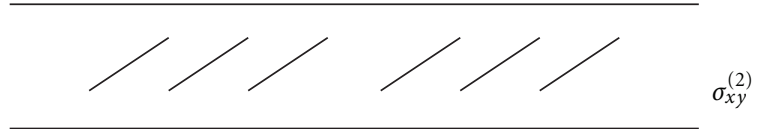

$\sigma_{x y}^{(1)}$

FIGURE 1: Layered media.

In other words, the Hall current cannot cross a phase boundary. It always flows round inhomogeneities, except for the finite number of singular points. Accordingly, for the first time it look like as that Hall conductivity in the heterogeneous medium always must be equal to zero. However, Hall conductivity is finite in reality.

The aim of present paper is to study the current percolation at the QHE conditions in the medium with boundaries. Values of Hall conductivity were found depending on conditions how the current percolates-along or across the layers. Also, the finite value of Hall conductivity at QHE conditions in media with boundaries was explained.

The structure of the paper is as follows. In the next section we consider a current percolation at QHE conditions in layered media. Section 3 is devoted to the current percolation in media, in which layers are shifted one with respect to the other. Within the conformal transformation method, local distributions of current and field were found in layered media, in which regions are shifted in a "chess order", black and white, black and white, and so on (see Figure 2). Short discussion of the obtained results is presented in Section 4.

\section{Current Percolation at QHE Conditions in the Layered Medium}

In order to understand the features of current percolation at QHE conditions let us consider a simple model, in which two layered periodic media have different Hall conductivities $\sigma_{x y}^{(1)}$ and $\sigma_{x y}^{(2)}$ [5] (see Figure 1). From theoretical point of view the different phases may be considered as different materials, which both display the quantum Hall effect and are connected as it has been shown in Figure 1, or they are from the same material with different degrees of local disorder.

2.1. Conductivity at QHE Regime When the Percolation Is Across the Layers. Let us consider the case when the electric current flows perpendicularly to the phase boundary. At QHE conditions, such a current assures the electric field to be directed along layers. From the continuity of tangential components it follows that the field is equal to the average value $E_{x}=\langle\vec{e}\rangle_{x}$.

Accordingly, we obtain

$$
\sigma_{x y}^{e}=\frac{1}{2}\left(\sigma_{x y}^{(1)}+\sigma_{x y}^{(2)}\right)
$$

Thus, the value of effective Hall conductivity (7) corresponds to solution with the constant electric field. In order to verify this result let us calculate the distribution of electric fields and currents in the layered media at the QHE regime. We use the definition of averages:

$$
\begin{gathered}
\langle\vec{e}\rangle_{1}+\langle\vec{e}\rangle_{2}=\vec{E}, \\
\sigma_{x y}^{(1)}\langle[\vec{n}, \vec{e}]\rangle_{1}+\sigma_{x y}^{(2)}\langle[\vec{n}, \vec{e}]\rangle_{2}=\sigma_{x y}^{e}[\vec{n}, \vec{E}]
\end{gathered}
$$

in order to obtain formulae describing the distribution of electric fields in phases:

$$
\langle\vec{e}\rangle_{1}=\vec{E} \frac{\sigma_{x y}^{e}-\sigma_{x y}^{(2)}}{\sigma_{x y}^{(1)}-\sigma_{x y}^{(2)}}, \quad\langle\vec{e}\rangle_{2}=\vec{E} \frac{\sigma_{x y}^{e}-\sigma_{x y}^{(1)}}{\sigma_{x y}^{(1)}-\sigma_{x y}^{(2)}} .
$$

By inserting the formula (8) in expressions (9), one can easily check that the solution (7) corresponds to constant electric field

$$
\langle e\rangle_{1 x}=\langle e\rangle_{2 x}=\frac{E_{x}}{2} .
$$

At first look, the obtained solution (7) for the Hall conductivity does not corresponds to new boundary conditions (6). According to these boundary conditions, Hall current cannot cross the phase boundaries, except for finite number of singular points. Thus, Hall conductivity must be equal to zero. Actually, the Hall currents flow along the phase boundaries and cross at infinity, which is the singular point. Percolation through the finite number of singular points (through infinity in our case) leads to nonzero value of the Hall conductivity.

2.2. Conductivity at $\mathrm{QHE}$ Conditions When the Percolation Is along the Layers. Below, we consider the current percolation along the layers and seek for the solution with the constant electric current. Then after averaging the currents we get an formula for the Hall conductivity

$$
\sigma_{y x}^{e}=\frac{2 \sigma_{x y}^{(1)} \sigma_{x y}^{(2)}}{\sigma_{x y}^{(1)}+\sigma_{x y}^{(2)}} .
$$

Inserting the formula (11) in the expressions for currents one can find that the solution (11) corresponds to constant electric current:

$$
\langle j\rangle_{1 y}=\langle j\rangle_{2 y}=\frac{\sigma_{x y}^{(1)} \sigma_{x y}^{(2)}}{\sigma_{x y}^{(1)}+\sigma_{x y}^{(2)}} E_{y}=J_{y} .
$$

Thus, the Hall conductivity in the layered systems has a tensor structure

$$
\hat{\sigma}_{x y}^{e}=\left(\begin{array}{cc}
0 & \sigma_{x y}^{e} \\
\sigma_{y x}^{e} & 0
\end{array}\right)
$$

We stress that the components of tensor of the Hall conductivity are not equal to each other:

$$
\sigma_{x y}^{e} \neq \sigma_{y x}^{e}
$$






FIGURE 2: Layered media with shifting of layers at the beginning of coordinates.

We note that obtained result is not in a contradiction with Onsager's principle of symmetry of kinetic coefficients, because this considered system is not isotropic and homogeneous since there is a preferential direction in the system direction of layers.

\section{Local Distributions and the Hall Conductivity of the Layered Systems with Shifted Layers at QHE Conditions}

In this section the layered systems with layers shifted with respect to each other at the origin have been considered (see Figure 2). This model has used in order to understand how the local fields and currents are distributed in the systems with the interphase boundaries at QHE conditions:

The systems with the interphase boundaries under Quantum Hall effect conditions.

3.1. The Methods of the Complex Variable Function Theory in the Two-Dimensional Problem of Conductivity. In order to find the local distribution of currents (fields) in layered media, in which regions are shifted in a chess order at the origin of coordinates, the methods of the complex variable function theory have been used. For this, let us consider the problem of conductivity in terms of the complex variable function theory [5-7].

Let us consider a plane of complex variable $z=x+i y$ and introduce the complex expressions for the two-dimensional electric currents and electric fields:

$$
j^{(k)}(z)=j_{x}^{(k)}-i j_{y}^{(k)}, \quad e^{(k)}(z)=e_{x}^{(k)}-i e_{y}^{(k)} .
$$

Here $k=1,2$ is a phase number. As is well known, such presentation is possible for the functions which satisfy Cauchy-Riemann conditions. Let us verify the validity of these conditions for the functions of electric currents and fields (15) introduced above. The continuity equation gives one of these conditions:

$$
\frac{\partial j_{x}^{(k)}}{\partial x}=-\frac{\partial j_{y}^{(k)}}{\partial y} .
$$

And the second condition follows from the equation $\operatorname{rot} \overrightarrow{\mathrm{e}}=$ 0 which is equivalent to the equation rot $\vec{j}=0$ for the media with a piecewise continuous conductivity:

$$
\frac{\partial j_{x}^{(k)}}{\partial y}=\frac{\partial j_{y}^{(k)}}{\partial x} .
$$

By the same way it is possible to check the Cauchy-Riemann conditions for the electric field.

The Ohm's law in the magnetic field can also be presented in the following form:

$$
j(z)=\frac{\sigma}{1-i \beta} E(z) .
$$

3.2. Conformal Mapping on a Z Plane. Due to biperiodic symmetry of the layered media it is enough to consider an elementary cell consisting of two adjacent semi-infinite stripes with different conductivities. Below, we construct the conformal mapping of the internal areas of adjacent semistripes with conductivities $\sigma_{1}, \sigma_{2}$ on the lower and upper semiplanes. In our case the following function represents such a transformation $[7,8]$ :

$$
\zeta=\cosh \left(\frac{\pi z}{L}\right)
$$

Here $\cosh (x)$ is hyperbolic cosine, $L$ is the width of the stripe. Conformal mapping leads to that the semistripe $a b c d$ has transformed into the lower semiplane, and semistripe $a b c^{\prime} d^{\prime}$ has transformed into the upper semiplane. The correspondence of the dots while mapping is shown in Figure 3.

3.3. Solution of the Boundary Problem in the Matrix Form. The boundary conditions are still valid for the electric current $j^{(k)}(\zeta)=j_{\xi}^{(k)}(\xi, \eta)-i j_{\eta}^{(k)}(\xi, \eta)$ in the plane $\zeta=\xi+i \eta$. They have different forms at different parts of axis $O \xi$ :

$$
j_{1}-\bar{j}_{1}=0, \quad j_{2}-\bar{j}_{2}=0
$$

at $L_{1}$ which includes rays $[b a),[c d),\left[c^{\prime} d^{\prime}\right)$. A dash above the variable means the complex conjugation

$$
j_{1}+\bar{j}_{1}=0, \quad j_{2}+\bar{j}_{2}=0
$$

at $L_{2}$ which includes segments $[b c],\left[b c^{\prime}\right]$.

Thus, the initial boundary conditions are reduced to the Riemann boundary problem for the piecewise continuous function $j(\zeta)$. The coefficients of the boundary problem have discontinuities in the axis $O \xi$ at points $b, c, c^{\prime}$. As it follows from the boundary conditions, there are four unknown functions in the problem: $j_{k}, \bar{j}_{k}, k=1,2$. In accordance with [5], we consider $j_{2}, \bar{j}_{1}$ to be functions of their complex conjugates $j_{2}, \bar{j}_{1}$. We introduce piecewise continuous vector function:

$$
\Phi(\zeta)=\left(\begin{array}{l}
j_{2}(\zeta) \\
\bar{j}_{1}(\zeta)
\end{array}\right) .
$$




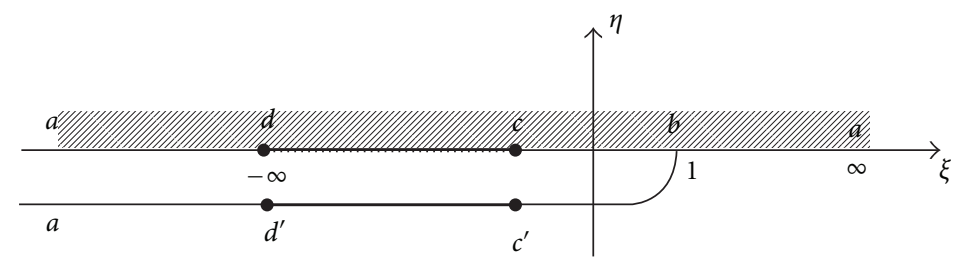

Figure 3: The conformal mapping: correspondence of the points at mapping.

This function satisfies the condition of symmetry:

$$
\bar{\Phi}(\bar{\zeta})=\left(\begin{array}{ll}
0 & 1 \\
1 & 0
\end{array}\right) \Phi(\zeta) .
$$

Accordingly, this function takes values in the $O \xi$ axis:

$$
\begin{aligned}
& \Phi^{+}(\xi)=\left(\begin{array}{l}
j_{2}(\xi) \\
\bar{j}_{1}(\xi)
\end{array}\right), \\
& \Phi^{-}(\xi)=\left(\begin{array}{l}
j_{1}(\xi) \\
\bar{j}_{2}(\xi)
\end{array}\right) .
\end{aligned}
$$

Thus, these boundary conditions lead to the Riemann boundary problem in the vector-matrix form:

$$
\Phi^{+}(\xi)=G_{i} \Phi^{-}(\xi)
$$

In the considered case of percolation at QHE conditions matrix coefficients have a simple form. At the interval $L_{1}$

$$
G_{1}=\left(\begin{array}{ll}
0 & 1 \\
1 & 0
\end{array}\right)
$$

and at the interval $L_{2}$

$$
G_{2}=\left(\begin{array}{cc}
0 & -1 \\
-1 & 0
\end{array}\right) .
$$

One can obtain the solution by reducing (25) to the system of two independent scalar Riemann boundary problems. For this, one needs to diagonalize the matrices of boundary conditions. Let us introduce a new piecewise continuous analytical function:

$$
\Psi(\xi)= \begin{cases}\Phi(\xi), & \text { if } \operatorname{Im}(\zeta)>0 \\ M \Phi(\xi), & \text { if } \operatorname{Im}(\zeta)<0\end{cases}
$$

At the interval $L_{1}$ there is a boundary condition for this function:

$$
\Psi^{+}(\xi)=\Psi^{-}(\xi)
$$

It means that the function has extended analytically at this interval.

Considering the interval $L_{2}$ we get

$$
\Psi^{+}(\xi)=G_{2} M^{-1} \Psi^{-}(\xi) .
$$

We introduce the other function:

$$
\Phi(\xi)=S F(\xi)
$$

Here the matrix $S$ has been used in order to diagonalize the matrix of boundary conditions at the interval $L_{2}$. As a result, we obtain the system of two independent Riemann boundary problems

$$
\Psi_{2}(\xi)=\lambda_{2} F_{2}(\xi)
$$

The eigen values have been determined from the equation:

$$
\left\|\lambda E-M^{-1} G_{2}\right\|=0 .
$$

Here $E$ is a unity matrix.

In the QHE case the eigen values have a simple form and are equal to

$$
\lambda_{1,2}=-1
$$

Accordingly, the solutions of scalar Riemann problems are given by $[7,8]$

$$
\begin{gathered}
F_{1}(\xi)=C_{1} X(\zeta), \quad F_{2}(\xi)=C_{2} X^{-1}(\zeta), \\
X(\xi)=\left[\frac{(\zeta-1)(\zeta+1)}{\zeta}\right]^{1 / 2} .
\end{gathered}
$$

Remember that the variable $\zeta$ has been connected with the initial variable $z$ through the formula (19). Further, we return to the initial vector function; we define the phases of coefficients $C_{1}, C_{2}$ too. As it follows from the symmetry conditions, the coefficients satisfy the relations:

$$
\frac{C_{1}}{\bar{C}_{1}}=\exp \left(i \frac{\pi}{2}\right), \quad \frac{C_{2}}{\bar{C}_{2}}=\exp \left(-i \frac{\pi}{2}\right) .
$$

Thus we obtain the expressions for local currents:

$$
\begin{gathered}
j_{1}(\zeta)=\left|C_{1}\right| \exp \left(i \frac{\pi}{4}\right) X(\zeta)=C_{1} X(\zeta), \\
j_{2}(\zeta)=\left|C_{2}\right| \exp \left(-i \frac{\pi}{4}\right) X^{-1}(\zeta)=C_{2} X^{-1}(\zeta) .
\end{gathered}
$$

Absolute values of coefficients $C_{1}, C_{2}$ are determined by external currents (fields). Namely,

$$
\begin{gathered}
\left\langle j_{1}\right\rangle+\left\langle j_{2}\right\rangle=J_{x}-i J_{y}=C_{1}\langle X(\zeta)\rangle+C_{2}\left\langle X^{-1}(\zeta)\right\rangle \\
\left\langle e_{1}\right\rangle+\left\langle e_{2}\right\rangle=E_{x}-i E_{y}=\rho_{x y}^{(1)} C_{1}\langle X(\zeta)\rangle+\rho_{x y}^{(2)} C_{2}\left\langle X^{-1}(\zeta)\right\rangle .
\end{gathered}
$$


Let us introduce the effective Hall conductivity tensor:

$$
\left(\begin{array}{l}
J_{x} \\
J_{y}
\end{array}\right)=\left(\begin{array}{cc}
0 & \sigma_{x y}^{e} \\
\sigma_{y x}^{e} & 0
\end{array}\right)\left(\begin{array}{l}
E_{x} \\
E_{y}
\end{array}\right) .
$$

According to the formulae (37)-(39), the components of the effective Hall conductivity tensor are given by

$$
\begin{aligned}
\sigma_{x y}^{e} & =\frac{I \operatorname{Re}\left(C_{1}+C_{2}\right)}{\operatorname{Im}\left(\rho_{x y}^{(1)} C_{1}+\rho_{x y}^{(2)} C_{2}\right)}, \\
\sigma_{y x}^{e} & =\frac{I \operatorname{Im}\left(C_{1}+C_{2}\right)}{\operatorname{Re}\left(\rho_{x y}^{(1)} C_{1}+\rho_{x y}^{(2)} C_{2}\right)} .
\end{aligned}
$$

Here $I=\langle X(\zeta)\rangle=\left\langle X^{-1}(\zeta)\right\rangle$.

Firstly, it is easy to see that the components of the Hall conductivity tensor are expressed in terms of constants $\left|C_{1}\right|,\left|C_{2}\right|$. They are not equal to each other in general:

$$
\sigma_{x y}^{e} \neq \sigma_{y x}^{e}
$$

Secondly, the values of constants $\left|C_{1}\right|,\left|C_{2}\right|$ are determined from conditions at infinity.

As an example, let us consider two types of conditions at infinity. by

(1) Assume the mean value of current at infinity is given

$$
J_{x}=\text { const }, \quad J_{y}=0 .
$$

We get then:

$$
\begin{gathered}
\left(\left|C_{1}\right|+\left|C_{2}\right|\right) 2 \cos \left(\frac{\pi}{4}\right) I=J_{x}, \\
\left(\left|C_{1}\right|-\left|C_{2}\right|\right) 2 \sin \left(\frac{\pi}{4}\right) I=J_{y}=0 .
\end{gathered}
$$

Therefore, it follows that

$$
\left|C_{1}\right|=\left|C_{2}\right|
$$

If these constants are equal to geometrical mean value

$$
\left|C_{1}\right|=\left|C_{2}\right|=\sqrt{\sigma_{x y}^{(1)} \sigma_{x y}^{(2)}}
$$

we obtain the following formula for the tensor component:

$$
\sigma_{x y}^{\perp}=\left\langle\rho_{x y}^{-1}\right\rangle=\frac{2 \sigma_{x y}^{(1)} \sigma_{x y}^{(2)}}{\sigma_{x y}^{(1)}+\sigma_{x y}^{(2)}} .
$$

This result corresponds to the solution with the direct electric current.

(2) Let us consider the second condition, when the electric field is defined at infinity. In this case we obtain

$$
\begin{gathered}
\left(\rho_{x y}^{(1)}\left|C_{1}\right|+\rho_{x y}^{(2)}\left|C_{2}\right|\right) 2 \sin \left(\frac{\pi}{4}\right) I=E_{x}, \\
\left(\rho_{x y}^{(1)}\left|C_{1}\right|-\rho_{x y}^{(2)}\left|C_{2}\right|\right) 2 \cos \left(\frac{\pi}{4}\right) I=E_{y}=0 .
\end{gathered}
$$

Therefore the following relation takes place:

$$
\rho_{x y}^{(1)}\left|C_{1}\right|=\rho_{x y}^{(2)}\left|C_{2}\right|
$$

If these constants are given by

$$
\left|C_{1}\right|=\frac{1}{\rho_{x y}^{(1)}}, \quad\left|C_{2}\right|=\frac{1}{\rho_{x y}^{(2)}}
$$

we obtain the following formula for the tensor component:

$$
\sigma_{x y}^{\perp}=\left\langle\sigma_{x y}\right\rangle=\frac{1}{2}\left(\sigma_{x y}^{(1)}+\sigma_{x y}^{(2)}\right) .
$$

This result corresponds to the solution with the constant electric field.

\section{Conclusions}

The obtained results for effective Hall conductivity are connected with unusual character of current percolation at QHE conditions. In this case, it follows from equation $\operatorname{div} \vec{j}=0$ and potentiality of electric field $\operatorname{rot} \vec{e}=0$ :

$$
\vec{e} \times \vec{\nabla} \sigma_{x y}=0,
$$

that is, the current lines never cross the isolines of quantity $\sigma_{x y}[4]$. In other words, the Hall current follows along lines of constant values of Hall conductivity and does not cross phase boundaries. It has "frozen" in each phase. This explains why the Hall phase as a function of phase concentrations has the plateau region. Moreover, the plateau value has been determined by conductivity of the percolating phase, which forms an infinite percolation cluster. It means that the plateau has been defined by the topology of current paths [ 9 , 10]. The current dependence on topology in quantum Hall effect and local characteristics have been studied too [11-14]. The current percolation under Quantum Hall conditions in the "chess-board"-like structures and distributions of local electric fields and currents have been studied also in [15-17].

As for nonzero value of the effective Hall conductivity at QHE conditions, we showed that it was due to the percolation of the Hall current through the finite number of singular points. We note that, as follows from our results, the conditions at infinity determine a character of percolation through singular points, which are corners at the phase joints in our model. There are two possibilities for the boundary Hall current percolation. First of them is the percolation of the boundary current from the first phase to the second one successively through the corner junctions. This result corresponds to the solution with the constant current at infinity (46). However, in the same system the other solution is also possible, when the average field is defined at infinity (50). In this case the boundary Hall currents flow separately, each along its phase and penetrate through the corners of semistripes located in a chess order.

Now the problem of construction of electron quantum interferometers is intensively studied due to a possibility of using these quantum devices for a study of many coherent inference phenomena $[18,19]$. As anolog of traditional 
optical interferometers the solid state interferometers, based on quantum Hall effect, are now constructed [20]. The main element of construction of such solid-state interferometers are the edge Hall currents. So the distribution of edge currents and conditions of percolation are very important for its construction. From this point of view the aboveobtained results will be useful for a construction and improvement of technique of solid-state quantum interferometers. Furthermore it is possible to construct a new type of two-phase heterogeneous solid-state interferometers, where the transitions between edge states will be determined by properties of singular points (corners at the phase joints).

The obtained results can also be used in the case of the strong magnetic field regime to find the Hall constant (which is determined by the carrier's concentration). According to our results, the effective Hall concentration at strong magnetic fields and the two-phase case have been determined by the concentration of the phase, which forms a percolation cluster. If the concentration is distributed continuously in some interval, the answer is that the effective Hall concentration in strong magnetic field regime has been determined by the concentration of carriers at the percolation threshold.

\section{Acknowledgment}

The research has partially been supported by Grant of Russsian Foundation for Basic researchers (Grant 10-0200573).

\section{References}

[1] J. B. Keller, "A theorem on the conductivity of a composite medium," Journal of Mathematical Physics, vol. 5, no. 4, pp. 548-553, 1964.

[2] A. M. Dykhne, "Conductivity of the two-dimensional diphasic environment," Journal of Experimental and Theoretical Physics, vol. 59, pp. 110-115, 1970 (Russian).

[3] A. M. Dykhne, "Abnormal resistance of plasma in a strong magnetic field," Journal of Experimental and Theoretical Physics, vol. 59, pp. 641-647, 1970 (Russian).

[4] A. M. Dykhne and I. M. Ruzin, "Theory of the fractional quantum Hall effect: the two-phase model," Physical Review B, vol. 50, no. 4, pp. 2369-2379, 1994.

[5] Yu. P. Emets, The Electric Descriptions of Composition Materials with the Regular Structure, The Naukova Dumka, Kiev, Russian, 1986.

[6] Yu. P. Emets, "Transformations of symmetry of twodimensional two-componental electrospending system," Journal of Experimental and Theoretical Physics, vol. 96, pp. 701710, 1989 (Russian).

[7] M. A. Lavrentiev and B. V. Shabat, Methods of the Theory of Functions of Complex Variable, Nauka, Moscow, Russia, 1973.

[8] A. I. Markushevich, The Short Course of the Theory of Analytical Functions, Nauka, Moscow, Russia, 1978.

[9] V. E. Arkhincheev, "About motionless points, invariants of transformations of Dyhne," Journal of Experimental and Theoretical Physics Letters, vol. 67, no. 2, pp. 951-958, 1998 (Russian).

[10] V. E. Arkhincheev and E. G. Batiyev, "On the theory of the quantum Hall effect in inhomogeneous medium," Solid State Communications, vol. 71, no. 12, pp. 1059-1060, 1989.
[11] V. E. Arkhincheev, "Bound values for Hall conductivity of heterogeneous medium under quantum Hall effect conditions," Pramana, vol. 70, no. 2, pp. 271-277, 2008.

[12] A. Alvermann and H. Fehske, "Stochastic Green's function approach to disordered systems," Journal of Physics: Conference Series, vol. 35, no. 1, pp. 145-156, 2006.

[13] J. E. Avron, D. Osadchy, and R. Seiler, "A topological look at the quantum hall effect," Physics Today, vol. 56, no. 8, pp. 3842, 2003.

[14] A. Forrester and Y. Joglekar, Conductivities in Bilayer Quantum Hall Systems, 2003.

[15] V. E. Arkhincheev, "Bound values for Hall conductivity and percolation under quantum Hall effect conditions," Physica $B$, vol. 403, no. 1, pp. 25-30, 2008.

[16] V. E. Arkhincheev, "Percolation in inhomogeneous medium under quantum Hall effect conditions," Physica A, vol. 285, no. 3, pp. 373-382, 2000.

[17] V. E. Arkhincheev, "Quantum hall effect in inhomogeneous media: effective characteristics and local current distribution," Journal of Experimental and Theoretical Physics, vol. 91, no. 2, pp. 407-415, 2000 (Russian).

[18] E. V. Deviatov and A. Lorke, "Separately contacted edge states at high imbalance in the integer and fractional quantum Hall effect regime," Physica Status Solidi B, vol. 245, no. 2, pp. 366377, 2008.

[19] E. V. Deviatov and A. Lorke, "Experimental realization of a Fabry-Perot-type interferometer by copropagating edge states in the quantum Hall regime," Physical Review B, vol. 77, no. 16, Article ID 161302, 2008.

[20] Y. Ji, Y. Chung, D. Sprinzak, M. Heiblum, D. Mahalu, and H. Shtrikman, "An electronic Mach-Zehnder interferometer," Nature, vol. 422, no. 6930, pp. 415-418, 2003. 

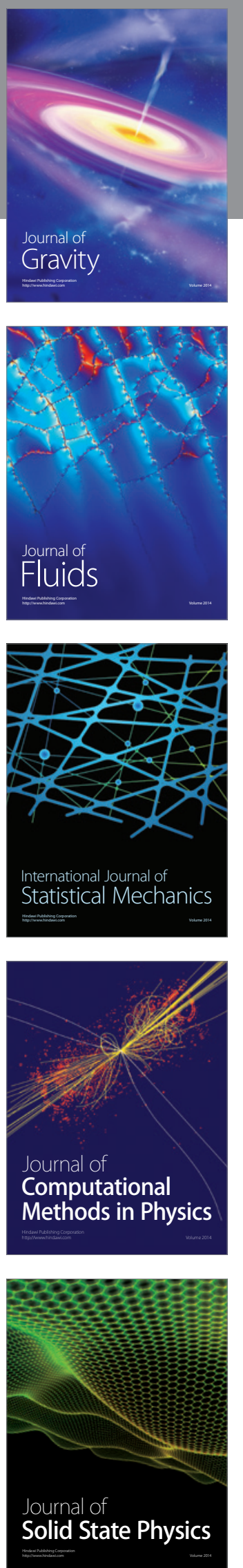

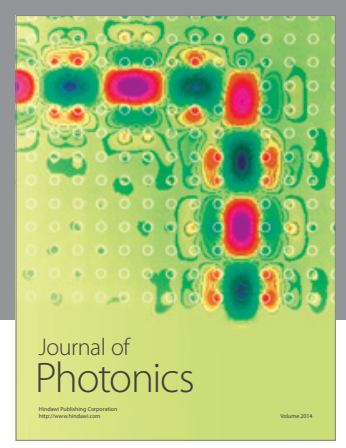



The Scientific World Journal
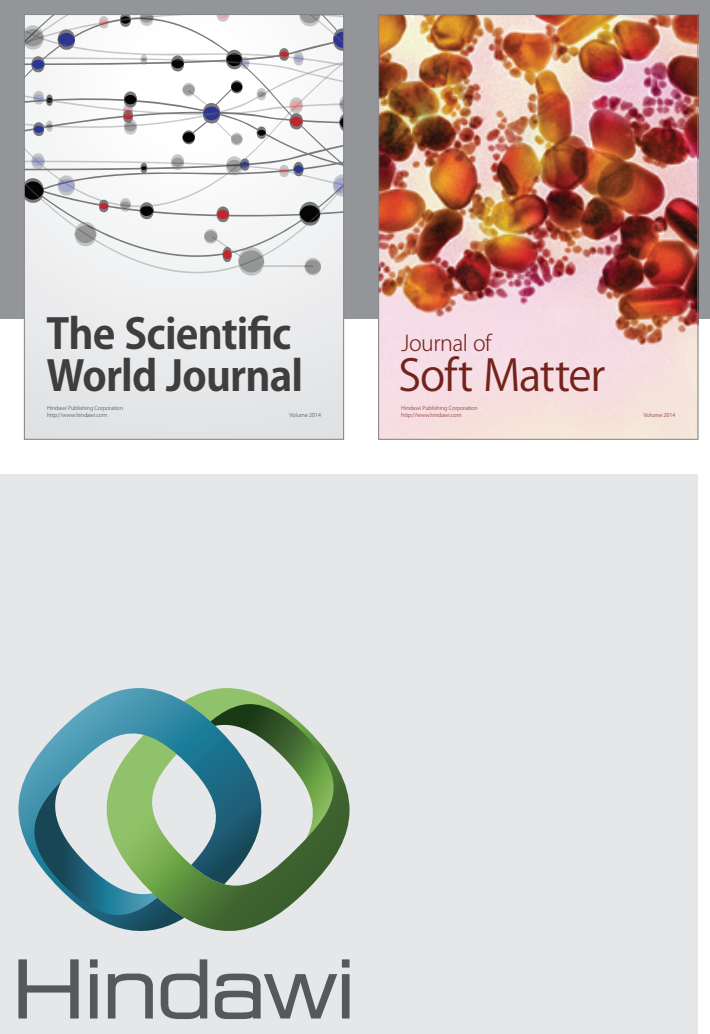

Submit your manuscripts at

http://www.hindawi.com
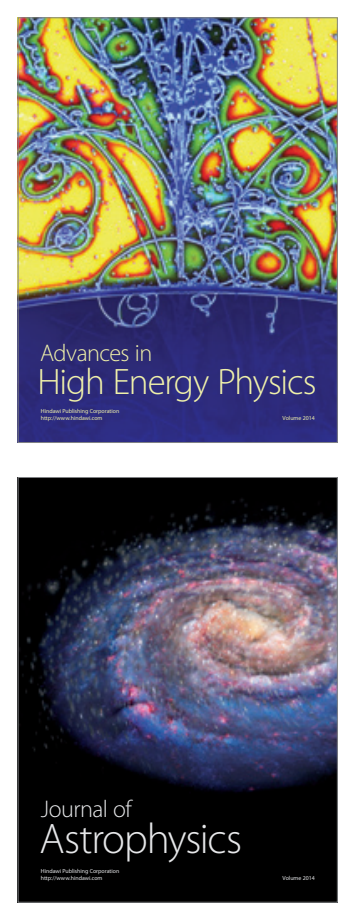
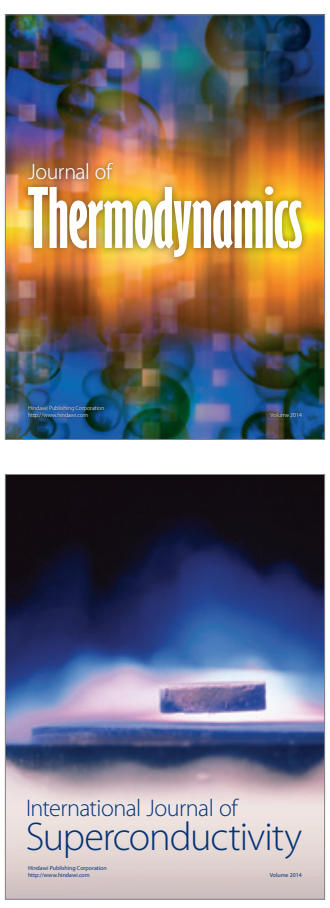
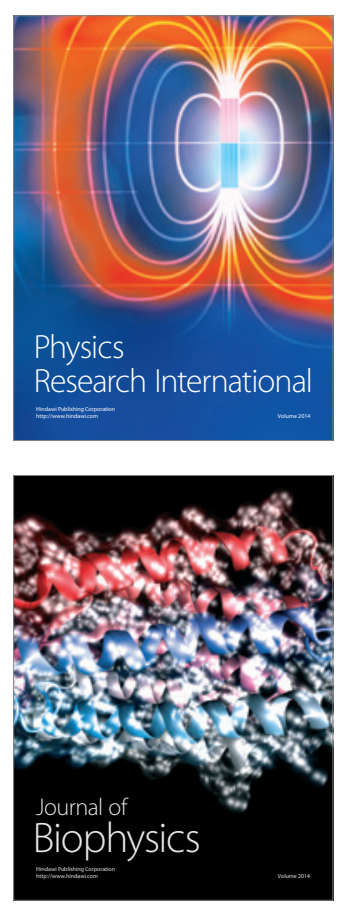
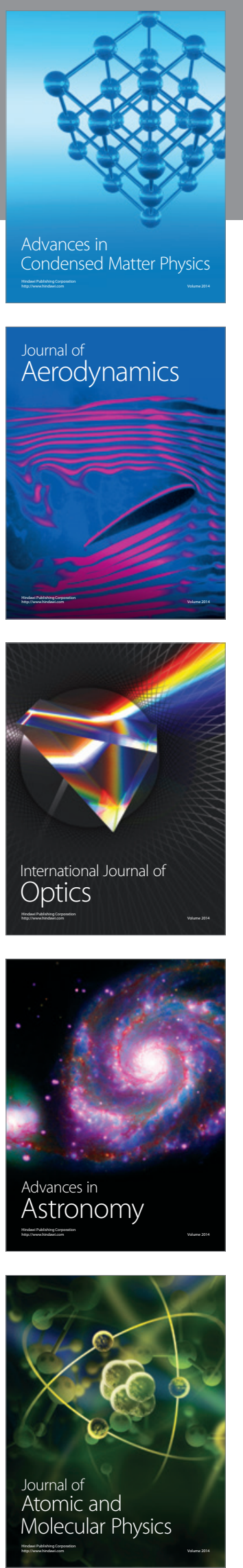